Journal of Animal and Veterinary Advances 11 (18): 3276-3280, 2012

ISSN: $1680-5593$

(C) Medwell Journals, 2012

\title{
Isolation and the Analysis of 16S rDNA Sequence of Swine Actinobacillus pleuropneumonia
}

\author{
Wan Hongping, Zuo Zhicai, Fu Tongchao, Dai Hongbo, Chen Lei, Xu Zhiwen and Zhu Lin \\ Sichuan Agricultural University, Ya’an, 625014 Sichuan, P.R. China
}

\begin{abstract}
A highly infectious and fatal respiratory tract disease occurred in a swine farm in Sichuan province. To explore the reason of the disease, samples of the liver and lung tissue, acquired sterilely in a typically sickened pig were intended for pathogenic detection. The suspicious strain, named after SCAB-1 was diagnosed which depended on cultural characteristics, morphologic observation and Nicotinamide Adenine Dinucleotide (NAD) experiment. Its pathogenicity was diagnosed by the virulence test on mice. And the strain was exactly identified by the analysis of $16 \mathrm{~S}$ rDNA sequence with its resistance inspected by sensitive test. According to cultural characteristics, morphology, NAD experiment and the analysis of $16 \mathrm{~S}$ rDNA sequence, it revealed that the study triumphantly isolated a strain of Actinobacillus pleuropneumonia which could lead a mouse to death, after infected $16 \mathrm{~h}$ and depend on NAD. The homology of the 16S rDNA sequence between SCAB-1 and Actinobacillus pleuropneumonia is highly 99.9\%. SCAB-1, Actinobacillus pleuropneumonia is susceptible to ceftriaxone, cefotaxime, cefuroxime, norfloxacin, SMZco; moderately sensitive to cefazolin, cefoperazone and resistance to cephaloridine IV, vancomycin and gentamycin.
\end{abstract}

Key words: Actinobacillus pleuropneumonia, pathogen isolate, $16 \mathrm{~S}$ rDNA, sensitive test, mouse

\section{INTRODUCTION}

Porcine contagious pleuropneumonia characterized by necrotizing bronchopneumonia and Fibrinous pleuritis is caused by Actinobacillus pleuropneumonia (App) (Chiers et al., 2010; Wang et al., 2010). The highly infectious and fatal respiratory tract disease has caused great economic losses throughout the worldwide swine industries (Li et al., 2012). The acute pleuropneumonia, mostly susceptible to pigs of approximately 10 weeks of age is featured by edema, hemorrhage, necrosis, hemorrhagic necrotic lesions in the lungs and pleural adhesions, leading to high mortality (Chiers et al., 2010; Subashchandrabose et al., 2009; Da Costa et al., 2004). App belongs to the Pasteurellaceae family which has been divided into biotype 1 strains which is dependent on Nicotinamide Adenine Dinucleotide (NAD) and biotype 2 strains which is independent of NAD (Archambault et al., 2012) and can grow on MacConkey agar plates. Whereas, the culture of biotype 1 strains faced many difficulties because of their fastidious growth characteristics. They cannot grow on ordinary agar plates or MacConkey agar plates but grow well on brain heart infusion agar plates, chocolate agar plates, sheep blood agar plates and Pleuropneumonia-Like Organisms (PPLO) agar plates because of their highly requirement of nutrition (Maldonado et al., 2009; Jacques et al., 2005;
He et al., 2003). The aim of the current study was to isolate a suspicious strain of App and identify it at both phenotypic and genotypic levels.

\section{MATERIALS AND METHODS}

$2 \times$ Taq PCR Master Mix, $\mathrm{ddH}_{2} \mathrm{O}$, DNA Marker DL2000, pMD19-T simple vecter, $\mathrm{T}_{4}$ DNA ligase, $10 \times$ Buffer for $\mathrm{T}_{4}$ DNA ligase, QIAquick Gel Extraction kit, Plasmid Extraction kit. Medicine sensitive pieces, biochemical tubes were purchased from Microbial Reagent Company (Hangzhou, China). DH5 $\alpha$ strains of Escherichia coli were kept by laboratory. Ten healthy $20 \mathrm{~g}$ mice, 4 healthy $2 \mathrm{~kg}$ rabbits without immunity for experiment and other material were provided by Sichuan Agricultural University.

Bacterial isolates and culture conditions: A highly infectious and fatal respiratory tract disease occurred in a swine farm in Sichuan province. Samples of the liver and lung tissue, acquired sterilely in a typically ill pig were intended for pathogenic detection. The aseptic samples were respectively grown on Tryptose Soya Agar (TSA) plates supplemented with $0.01 \%$ NAD, PPLO agar plates and chocolate agar plates. Plates were incubated at $37^{\circ} \mathrm{C}$ in $5 \% \mathrm{CO}_{2}$ for $24-48 \mathrm{~h}$. Colony, characterized by ivory, roundness and wee were extracted to purified cultivation.

Corresponding Author: Zhu Lin, Sichuan Agricultural University, Ya'an, 625014 Sichuan, P.R. China 
The isolated and purified suspicious strain, named after SCAB-1 which was added $40 \%$ glycerin was maintained at $-70^{\circ} \mathrm{C}$.

Cultural characteristics test: The isolates were respectively cultivated on ordinary agar plates, MacConkey agar plates, TSA plates and chocolate agar plates at $37^{\circ} \mathrm{C}$ in $5 \% \mathrm{CO}_{2}$ for $24-48 \mathrm{~h}$ with the different growing results on plates observed and recorded.

Morphologic observation: To observe the morphology of the isolates, a solo purified strain was extracted to Gram stain.

NAD experiment: The isolates were respectively cultured on PPLO agar plates containing $0.01 \%$ NAD and PPLO agar plates without $\mathrm{NAD}$ at $37^{\circ} \mathrm{C}$ in $5 \% \mathrm{CO}_{2}$ for $24-48 \mathrm{~h}$ to observe and record the results.

The virulence test on mice: Ten mice were randomly divided into two groups, one of the group being a contrast. The virulence of the isolates were measured by injecting $0.2 \mathrm{~mL}$ bacterium suspension using PBS to dilute to $5 \times 10^{8} \mathrm{CFU} \mathrm{mL}^{-1}$ in each mice of test group and $0.2 \mathrm{~mL}$ TSB without any bacterium to each mice of contrast group. The two groups were raised separately, the occurrence of disease being observed at regular time. The dead mice were instantly dissected with the pathological variation surveyed. The samples of liver and lung tissue of the dead mice were acquired sterilely to identify the pathogens.

The analysis of 16S rDNA sequence: Preparation for DNA template and Amplification for $16 \mathrm{~S}$ rDNA by PCR. $50 \mu \mathrm{L}$ bacterium and $100 \mu \mathrm{L}$ PBS buffer were blended in a spotless EP tube, boiled for $10 \mathrm{~min}$ and centrifuged for $1 \mathrm{~min}$ at $13000 \mathrm{r} \mathrm{min}^{-1}$. The supernatant were collected and maintained at $-20^{\circ} \mathrm{C}$ and subsequently were used for PCR gene amplification. A partial fragment about $1500 \mathrm{bp}$ of the $16 S r R N A$ gene was amplified using the universal primers for SCAB-1: 27F 5'-AGAGTTTGATCCTGG CTCAG-3' and 1492R 5'- GGTTACCTTGTTACGACTT-3 (Bal and Bal, 2012). PCR was conducted in the volume of $20 \mu \mathrm{L}$ total: $1 \mu \mathrm{L}$ of DNA template, $10.0 \mu \mathrm{L}$ of $2 \times \mathrm{PCR}$ Master Mix, $8.0 \mu \mathrm{L}$ of $\mathrm{ddH}_{2} \mathrm{O}, 0.5 \mu \mathrm{L}$ of each primer $(20 \mu \mathrm{L}$ of total). Amplification conditions were as follows: an initial denaturation step at $95^{\circ} \mathrm{C}$ for $5 \mathrm{~min}$ followed by 30 cycles ofdenaturation at $95^{\circ} \mathrm{C}$ for $35 \mathrm{sec}$, annealing at $55^{\circ} \mathrm{C}$ for $40 \mathrm{sec}$, polymerization at $72^{\circ} \mathrm{C}$ for $1.5 \mathrm{~min}$ and a final extension at $72^{\circ} \mathrm{C}$ for $7 \mathrm{~min}$. Amplified bands were separated by gel electrophoresis (1\% agarose gel) and visualized on a gel documentation system.
Purification of the PCR products and DNA sequencing and homology analysis: Based on the manufacturer's instructions, the QIAquick Gel Extraction kit was used to collect PCR products which were subsequently connected with pMD19-T simple vector with the compound transferred into $\mathrm{DH} 5 \alpha$ competent cells. The transferred strains incubated at $37^{\circ} \mathrm{C}$ in oscillation box for $1 \mathrm{~h}$ were grown on Amp plates coating method at $37^{\circ} \mathrm{C}$ for $12-24 \mathrm{~h}$, subsequently the transferred strains extracted to LB broth containing Amp to amplification. Plasmid Extraction kit was employed to extract recombinant plasmid, identified by PCR. The positive recombinant plasmid were confirmed by sequencing. The sequencing results of nucleotide sequences were compared with known sequences on Genbank using the BLAST. Then, homology of sequences was analysed and a phylogenetic tree was constructed via DNAStar Software.

Sensitive test: Ceftriaxone, cefotaxime, cefuroxime, norfloxacin, SMZco, cefazolin, cefoperazone, cephaloridine IV, vancomycin, gentamycin were employed to test the sensitivity of the purified strains about medicines judging by diameter inhibition zone.

\section{RESULTS AND DISCUSSION}

Cultural characteristics, morphology, NAD experiment and virulence: $\mathrm{SCAB}-1$ cannot grow on ordinary agar plates or MacConkey agar plates but it can grow on chocolate agar plates characterized by nearly roundness, uplift, hoar, tidy edge and wee and TSA agar plates containing $0.01 \% \mathrm{NAD}$ featured by ivory, circle and tip size. Meanwhile, SCAB-1 is a Gram-negative with the following traits: suborbicular, wee, single, consistent morphology without flagella and spore, unconspicuous dyeing both of ends which cannot be cultivated on PPLO agar plates without NAD but on PPLO agar plates containing $0.01 \% \mathrm{NAD}$. It can deduce that the growth of SCAB-1 strictly depends on NAD. On the other hand, mice of the test group were dead within $16 \mathrm{~h}$ however, mice of the contrast group survived. Inflammatory edema was discovered in injected subcutaneous tissue. It conclude that SCAB-1 is virulent.

DNA sequencing and homology analysis: The PCR product, purpose gene is corresponding to $1,500 \mathrm{bp}$ which is the expected length (Fig. 1).

According to the BLAST, the homology of the 16S rDNA sequence between SCAB-1 and Actinobacillus is high. Further comparision between SCAB-1 and fourteen gram negative bacterium via DNAStar Software 
Table 1: The homologous comparision of the $16 \mathrm{~S} r D N A$ gene between $\mathrm{SCAB}-1$ and fourteen gram negative bacterium

\begin{tabular}{|c|c|c|c|c|c|c|c|c|c|c|c|c|c|c|c|}
\hline Species & $\mathrm{s} 1$ & 2 & 3 & 4 & 5 & 6 & 7 & 8 & 9 & 10 & 11 & 12 & 13 & 14 & 15 \\
\hline 1 & - & 99.5 & 73.9 & 72.3 & 85.2 & 85.4 & 91.5 & 92.2 & 84.5 & 84.7 & 92.5 & 92.5 & 84.5 & 83.1 & 99.4 \\
\hline 2 & 0.1 & - & 74.4 & 72.8 & 85.6 & 85.8 & 92.0 & 92.7 & 84.9 & 85.1 & 93.0 & 93.0 & 84.9 & 83.6 & 99.9 \\
\hline 3 & 21.3 & 21.2 & - & 99.4 & 74.4 & 77.5 & 75.9 & 72.9 & 76.4 & 77.5 & 72.6 & 72.9 & 74.7 & 73.9 & 74.2 \\
\hline 4 & 21.6 & 21.5 & 0.0 & - & 73.3 & 76.4 & 75.2 & 72.3 & 76.6 & 76.4 & 71.6 & 71.6 & 73.8 & 73.6 & 72.7 \\
\hline 5 & 12.9 & 12.9 & 20.6 & 21.3 & - & 98.6 & 85.6 & 84.6 & 94.2 & 95.3 & 84.9 & 85.2 & 97.1 & 97.0 & 85.5 \\
\hline 6 & 12.6 & 12.6 & 20.2 & 20.8 & 0.7 & - & 86.0 & 85.4 & 93.6 & 95.7 & 85.6 & 85.9 & 96.3 & 96.4 & 85.8 \\
\hline 7 & 6.6 & 6.5 & 20.6 & 20.9 & 13.0 & 12.5 & - & 98.8 & 85.0 & 85.1 & 93.6 & 93.6 & 85.6 & 86.0 & 91.8 \\
\hline 8 & 6.7 & 6.5 & 20.8 & 21.0 & 12.9 & 12.4 & 0.5 & - & 84.9 & 85.0 & 94.3 & 94.4 & 84.6 & 85.0 & 92.5 \\
\hline 9 & 13.3 & 13.1 & 21.8 & 21.0 & 4.6 & 5.3 & 13.5 & 13.4 & - & 97.9 & 84.7 & 83.3 & 95.5 & 95.3 & 84.9 \\
\hline 10 & 13.3 & 13.1 & 20.5 & 21.1 & 3.5 & 4.0 & 13.5 & 13.3 & 1.4 & - & 85.2 & 85.6 & 95.5 & 95.3 & 85.1 \\
\hline 11 & 6.9 & 6.7 & 21.5 & 22.1 & 13.0 & 12.8 & 5.2 & 5.1 & 13.3 & 12.9 & - & 99.9 & 85.1 & 84.4 & 92.8 \\
\hline 12 & 6.9 & 6.7 & 21.1 & 21.9 & 12.7 & 12.4 & 5.1 & 5.0 & 14.5 & 12.7 & 0.1 & - & 85.1 & 84.4 & 92.8 \\
\hline 13 & 13.6 & 13.4 & 20.3 & 20.9 & 2.5 & 3.1 & 13.1 & 13.1 & 3.7 & 3.7 & 13.1 & 13.1 & - & 99.4 & 84.9 \\
\hline 14 & 13.9 & 13.7 & 20.6 & 20.7 & 2.4 & 3.1 & 13.2 & 13.2 & 3.9 & 3.9 & 13.8 & 13.8 & 0.4 & - & 83.6 \\
\hline 15 & 0.3 & 0.1 & 21.4 & 21.7 & 13.0 & 12.7 & 6.6 & 6.7 & 13.2 & 13.2 & 6.9 & 6.9 & 13.5 & 13.8 & - \\
\hline
\end{tabular}

1: Actinobacillus pleuropneumonia NR044752; $:$ Actinobacillus pleuropneumonia AF03305; $3:$ B. bronchiseptica X $57026 ; 4:$ Bordetella bronchiseptica NR_025949; 5: E. coli EHEC strain ATCC43895 Z83205; 6: E. coli strain KCTC 2441 EU014689; 7: H. parasuis FJ667962; 8: H. parasuis FJ667982; 9: Klebsiella DQ831003; 10: Klebsiella EU545402; 11: Pasteurella FJ405340; 12: Pasteurella multocida AF224297; 13: Salmonella enterica AF227869; 14: Salmonella enterica AF332600; 15: SCAB-1

Table 2: The result of the sensitive test

\begin{tabular}{lcl}
\hline Drugs name & Diameter of inhibition (mm) & Results \\
\hline Ceftriaxone & 22 & Susceptible \\
SMZco & 21 & Susceptible \\
Cefoperazone & 12 & Moderately sensitive \\
Cefotaxime & 19 & Susceptible \\
Cephaloridine IV & 3 & Resistance \\
Cefuroxime & 20 & Susceptible \\
Gentamycin & - & Resistance \\
Norfloxacin & 22 & Susceptible \\
Vancomycin & - & Resistance \\
Cefazolin & 8 & Moderately sensitive \\
\hline
\end{tabular}

indicate that the identity of the $16 \mathrm{~S}$ rDNA sequence between SCAB-1 and Actinobacillus pleuropneumonia is highly $99.9 \%$ while others is lower (Table 1).

A phylogenetic tree was constructed based on Clustal $\mathrm{W}$ through DNAStar Software, revealing that SCAB-1 and Actinobacillus pleuropneumonia were on a same branch (Fig. 2). It deduce that SCAB-1 belongs to Actinobacillus pleuropneumonia.

Sensitive test: Sensitive test showed that SCAB-1, Actinobacillus pleuropneumonia is susceptible to ceftriaxone, cefotaxime, cefuroxime, norfloxacin, SMZco; moderately sensitive to cefazolin, cefoperazone and resistance to cephaloridine IV, vancomycin, gentamycin (Table 2).

Actinobacillus pleuropneumonia (App) has caused porcine respiratory diseases which have heavily impacted the economy of the swine rearing industry worldwide (Auger et al., 2009). The App isolates could grow on PPLO agar plates, blood agar plates and chocolate agar plates but it is essential to supplement some antibacterial to inhibit the growth of other bacterium (He et al., 2003). During the isolation, the research faced many difficulties presented by the fastidious growth characteristics and emerging of nontypable isolates. The study laboriously isolated a strain on TSA agar plates supplied with $0.01 \%$

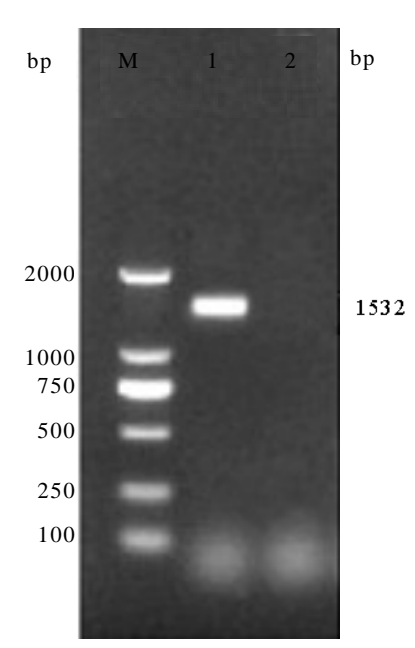

Fig. 1: Electrophoresis figure of $16 \mathrm{~S}$ rDNA by PCR; $\mathrm{M}=$ Molar mass marker, $1=$ PCR product, $2=$ Negative contrast

NAD suggesting that it is necessary to provide $5-10 \%$ $\mathrm{CO}_{2}$ to cultivate first isolates for 24-48 h. The study sterilely collected the lesion typical liver and lung tissue and then samples were recovered and cultured on TSA agar plates, PPLO agar plates and chocolate agar plates, finally with a suspicious strain, named after SCAB-1 was isolated from the samples. Through cultural characteristics, morphology, NAD experiment, virulence test, the research firstly consider that it maybe belong to Actinobacillus. Now a days, Molecular Phylogenetic Methods have revolutionized the classifying and identification of organisms that occur in microbial communities (Raje et al., 2010). The 16S rDNA gene is widely used to investigate the evolutionary relationships of prokaryotes. 


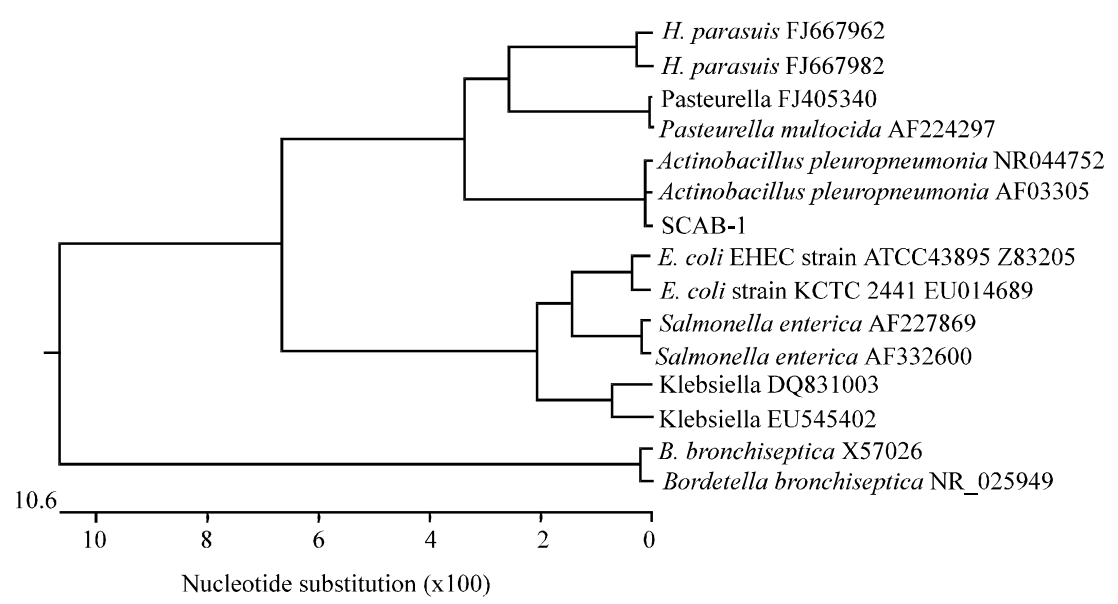

Fig. 2: The analysis of the $16 S$ gene phylogenesis. Multiple sequence alignment of the $16 S r D N A$ partial genes from strains analyzed in the study. Multiple alignment was done by the CLUSTAL program and used to construct the tree by the NJ Method

\section{CONCLUSION}

Based on the DNA sequencing, homology analysis and phylogenetic tree which showed that identity of $16 \mathrm{~S}$ $r D N A$ gene between SCAB-1 and Actinobacillus pleuropneumonia is highly $99.9 \%$, the study concludes that researchers triumphantly isolate a strain of Actinobacillus pleuropneumonia which is dependent on NAD. And it is susceptible to ceftriaxone, cefotaxime, cefuroxime, norfloxacin, SMZco; moderately sensitive to cefazolin, cefoperazone and resistance to cephaloridine $\mathrm{IV}$, vancomycin, gentamycin. It proposes that several medicines should being utilized alternately to avoid abusing of antibiotics. Furthermore, measures such as sanitation and exploitation of vaccine are entailed to defense the disease.

\section{ACKNOWLEDGEMENT}

Wan Hongping and Zuo Zhicai are contributed equally to the research.

\section{REFERENCES}

Archambault, M., J. Harel, J. Goure, Y.D.N. Tremblay and M. Jacques, 2012. Antimicrobial susceptibilities and resistance genes of canadian isolates of Actinobacillus pleuropneumoniae. Vet. Microb., 18: 198-206.

Auger, E., V. Deslandes, M. Ramjeet, I. Contreras and J.H.E. Nash et al., 2009. Host-pathogen interactions of Actinobacillus pleuropneumoniae with porcine lung and tracheal epithelial cells. Infec. Immun., 77: 1426-1441.
Bal, E.B.B. and M.A. Bal, 2012. Effects of chemical additives and ensiling time on whole plant wheat silage microbial pro?les inferred by phenotypic and $16 \mathrm{~S}$ ribosomal DNA analyses. World J. Microbiol. Biotechnol., 28: 767-776.

Chiers, K., T. De Waele, F. Pasmans, R. Ducatelle and F. Haesebrouck, 2010. Virulence factors of Actinobacillus pleuropneumoniae involved in colonization, persistence and induction of lesions in its porcine host. Vet. Res., Vol. 41. 10.1051/vetres/ 2010037.

Da Costa, M.M., R. Balestrin, A. Schrank, I.A. Piffer, S.C. Da Silva and I.S. Schrank, 2004. Evaluation of PCR based on gene apxIVA associated with 16S rDNA sequencing for the identification of Actinobacillus pleuropneumoniae and related species. Curr. Microbiol., 48: 189-195.

He, Q.G., G.P. Wang, J.F. Liu, H.C. Chen and H.H. Zhou et al., 2003. Isolation and identification Swine Actinobacillus pleuropneumonia and medicine sensitive test. Chin. J. Preventive Ver. Med., 25: $360-363$

Jacques, M., J. Labrie, F. St Michael, A.D. Cox and M.A. Paradis et al., 2005. Isolation of an atypical strain of Actinobacillus pleuropneumoniae serotype 1 with a truncated lipopolysaccharide outer core and no O-antigen. J. Clin. Microbiol., 43: 3522-3525.

Li, T., Z. Xu, T. Zhang, L. Li, H. Chen and R. Zhou, 2012. The genetic analysis of the flp locus of Actinobacillus pleuropneumoniae. Arch. Microbiol., 94: 167-176. 
Maldonado, J., L. Valls, E. Martinez and P. Riera, 2009. Isolation rates, serovars and toxin genotypes of nicotinamide adenine dinucleotide-independent Actinobacillus pleuropneumoniae among pigs suffering from pleuropneumonia in Spain. J. Vet. Diagn. Invest., 21: 854-857.

Raje, D.V., H.J. Purohit, Y.P. Badhe, S.S. Tambe and B.D. Kulkarni, 2010. Self-organizing maps: A tool to ascertain taxonomic relatedness based on features derived from 16S rDNA sequence. J. Biosci., 35: 617-627.
Subashchandrabose, S., R.M. LeVeque, T.K. Wagner, R.N. Kirkwood, M. Kiupel and M.H. Mulks, 2009. Branched-chain amino acids are required for the survival and virulence of Actinobacillus pleuropneumoniae in Swine. Infec. Immun., 77: 4925-4933.

Wang, Y.C., J.P.W. Chan, K.S. Yeh, C.C. Chang and S.L. Hsuan et al., 2010. Molecular characterization of enrofloxacin resistant Actinobacillus pleuropneumoniae isolates. Vet. Microbiol., 142: 309-312. 